\title{
Neutrophil-lymphocyte ratio and platelet-lymphocyte ratio associations with heart and body dose and their effects on patient outcomes in locally advanced non-small cell lung cancer treated with definitive radiotherapy
}

\author{
Wu-Yan Xia^, Xue-Ru Zhu, Wen Feng, Jun Liu, Jia-Ming Wang, Chang-Xing Lv, Qin Zhang, Wen Yu, \\ Xu-Wei Cai, Xiao-Long Fu^ \\ Department of Radiation Oncology, Shanghai Chest Hospital, Shanghai Jiao Tong University, Shanghai, China \\ Contributions: (I) Conception and design: WY Xia, XL Fu; (II) Administrative support: XL Fu; (III) Provision of study materials or patients: WY Xia, \\ J Liu, JM Wang, CX Lv, Q Zhang, W Yu, XW Cai, XL Fu; (IV) Collection and assembly of data: WY Xia, XR Zhu, XL Fu; (V) Data analysis and \\ interpretation: WY Xia, XL Fu; (VI) Manuscript writing: All authors; (VII) Final approval of manuscript: All authors. \\ Correspondence to: Xiao-Long Fu. Department of Radiation Oncology, Shanghai Chest Hospital, Shanghai Jiao Tong University, No. 241 West \\ Huaihai Road, Shanghai 200030, China. Email: xlfu1964@hotmail.com.
}

Background: Inflammation plays a vital role in tumor growth and progression and can be affected by radiotherapy (RT) and chemotherapy. We sought to investigate the prognostic significance of neutrophilto-lymphocyte ratio (NLR) and platelet-to-lymphocyte ratio (PLR), and their associations with dosimetric factors in locally advanced non-small cell lung cancer (LA-NSCLC).

Methods: In this retrospective study, subjects consisted of 244 patients who had received definitive RT \pm chemotherapy for LA-NSCLC between 2012 and 2016. Absolute lymphocyte count (ALC), NLR and PLR recorded at pretreatment, during RT and post-RT were analyzed. Multivariable analysis (MVA) was performed to correlate clinical factors and inflammatory biomarkers with progression-free survival (PFS) and overall survival (OS) using a Cox regression model. Relationships between NLR or PLR with OS and PFS were evaluated with Kaplan-Meier analysis and compared with log-rank test results. Multiple stepwise linear regression was used to assess the associations between dosimetric factors and NLR or PLR.

Results: The median PFS and OS for all patients were 8.6 and 15.8 months, respectively. On MVA for PFS and OS, higher 1-month post-RT start NLR [hazard ratio (HR) 1.049; 95\% CI: 1.018-1.080; P=0.001] or higher 1-month post-RT start PLR (HR 1.001; 95\% CI: 1.000-1.002; $\mathrm{P}<0.001$ ) was associated with inferior PFS. Higher 1-month post-RT start NLR (HR 1.040; 95\% CI: 1.013-1.069; P=0.004) or PLR (HR 1.001; 95\% CI: 1.001-1.002; $\mathrm{P}<0.001)$ was also an independent predictor of OS. ALCmin, baseline NLR and PLR were not associated with treatment outcomes. Multiple stepwise linear regression analysis confirmed that baseline NLR $(\mathrm{P}<0.001)$, heart V20 $(\mathrm{P}<0.001)$, heart V40 $(\mathrm{P}<0.001)$, and mean body dose (MBD) were significantly associated with 1 -month post-RT start NLR. Also, baseline PLR $(\mathrm{P}<0.001)$ and $\mathrm{MBD}(\mathrm{P}<0.001)$ were significantly associated with 1 -month post-RT start PLR.

Conclusions: Higher NLR and PLR during treatment were associated with worse patient outcomes, and heart dose or body dose was correlated with NLR or PLR in advanced NSCLC patients treated with definitive RT.

Keywords: Neutrophil-to-lymphocyte ratio (NLR) and platelet-to-lymphocyte ratio (PLR); heart dose; mean body dose (MBD); radiotherapy (RT); NSCLC

Submitted May 26, 2020. Accepted for publication Aug 14, 2020.

doi: $10.21037 /$ tlcr-20-831

View this article at: http://dx.doi.org/10.21037/tlcr-20-831

^ ORCID: Wu-Yan Xia: 0000-0001-5453-9034; Xiao-Long Fu: 0000-0001-8127-3884. 


\section{Introduction}

Locally advanced non-small cell lung cancer (NSCLC) (stage III) accounts for approximately $20-25 \%$ of patients with NSCLC at diagnosis (1). The main progress of the treatment for NSCLC includes immunotherapy and targeted therapy. Historically, the standard treatment for patients with unresectable locally advanced NSCLC (LA-NSCLC) was platinum-based concurrent chemoradiotherapy (2). However, prognosis has been poor, with a 5 -year overall survival (OS) rate ranging from $15 \%$ to $25 \%$, and the median OS time only $17-24$ months $(3,4)$. Durvalumab, an anti-programmed death ligand 1 (PD-L1) antibody, has been used in patients who have completed concurrent chemoradiation for inoperable LA-NSCLC. The immunotherapy after concurrent chemoradiation has been shown to significantly increase the tumor response rate, progression-free survival, and OS (5). Currently, durvalumab has become standard treatment for inoperable LA-NSCLC. The 2019 American Society of Clinical Oncology (ASCO) Annual Conference, revealed that the 36 -month OS rate was $57 \%$ in patients who received durvalumab as consolidation therapy after concurrent chemoradiation (6). Therefore, studying changes in inflammatory biomarkers during concurrent chemoradiation might be useful for optimizing the implementation of radiotherapy (RT) in the immunotherapy era.

Emerging evidence has indicated that systemic inflammation plays a vital role in the development and progression of many solid tumors $(7,8)$. The systemic inflammation, as determined by parameters such as lymphocytes, neutrophil-to-lymphocyte ratio (NLR), and platelet-to-lymphocyte ratio (PLR), correlate with prognosis in many malignancies, including NSCLC (9-13). For example, in lung cancer, elevated pretreatment NLR and PLR are associated with poor survival in patients with advanced NSCLC, especially for unresectable patients (14). However, there are relatively few studies on the correlation between the dynamic changes of these prognostic biomarkers and clinical outcomes.

Radiation is known to induce immunosuppression via direct destruction of mature circulating lymphocytes (15). Many researchers have explored radiation-induced lymphopenia in a variety of carcinomas $(16,17)$. Dosimetric analyses in NSCLC have demonstrated that lymphopenia is associated with lung V5 and gross tumor volume (GTV) (9). Since a large volume of blood circulates through the heart during thoracic radiation, we hypothesized that heart dose might be a predictive parameter of NLR and PLR during treatment. Fang et al. demonstrated that in patients with esophageal cancer, mean body dose (MBD) was inversely correlated with lymphocyte nadir (18). Therefore MBD may also be a predictive parameter of NLR and PLR during treatment. At present, no studies investigate the correlation between MBD and NLR or PLR during treatment in LANSCLC.

In this retrospective study, we present our investigation of patients with LA-NSCLC who received definitive radiation, and the impact of NLR and PLR during treatment on clinical outcomes. We further investigated the correlation between heart dose, MBD, NLR, and PLR during treatment. We present the following article in accordance with the STROBE reporting checklist (available at http://dx.doi.org/10.21037/tlcr-20-831).

\section{Methods}

\section{Patients}

NSCLC patients treated with definitive RT with or without chemotherapy from 2012 to 2016 at Shanghai Chest Hospital were retrospectively reviewed. Inclusion criteria included: pathologic confirmation of NSCLC with clinical stage III disease according to the TNM criteria of NSCLC (Union for International Cancer Control, eighth edition), receipt of a total radiation dose of at least $50 \mathrm{~Gy}$, documentation of RT dosimetric data and pretreatment and 1-month post-RT start complete blood counts (CBCs) (defined as the blood count obtained one month after the RT start). Exclusion criteria consisted of receipt of epidermal growth factor receptor tyrosine kinase inhibitor (EGFR-TKI) or anaplastic lymphoma kinase tyrosine kinase inhibitor (ALK-TKI) as neoadjuvant or adjuvant therapy for $\mathrm{RT}$, having an autoimmune disease, dying within 4 weeks after completion of RT.

\section{Data collection and follow-up}

Patient characteristics and CBCs data were obtained from electronic medical records. RT dose, GTV, MBD, heart, and lung dose were collected directly from the treatment plans for the patients. Variables from blood count included absolute lymphocyte count (ALC), NLR, and PLR. The most recent blood count before the initial point of commencement of definitive treatment (RT, chemotherapy, or both) was defined as baseline count. The 1-month post- 
RT count was defined as RT count obtained one month after the completion of RT. The NLR was calculated by dividing the absolute neutrophil count by the ALC (NLR = absolute neutrophil count/absolute lymphocyte count), and the PLR was calculated as the absolute platelet count divided by the ALC (PLR = absolute platelet count/absolute lymphocyte count). The ALCmin was defined as the minimum ALC during treatment.

Follow-up data was obtained retrospectively through the electronic medical records according to the standard practice of the disease. After treatment, patients were followed up every 3 months for the first 2 years, every 6 months from 3 to 5 years, and after that annually. The physician follow-up included clinical assessments, thoracic computed tomography (CT) scans, abdomen B-ultrasound examination, and other examinations as needed. Additional imaging, including brain magnetic resonance imaging (MRI), was obtained based on symptoms. The time of subsequent EGFR-TKI or ALK-TKI therapy for patients with the progressive disease was recorded. Patient followup was updated and censored on September 12, 2019.

All procedures performed in this study involving human participants were in accordance with the Declaration of Helsinki (as revised in 2013). The study was approved by the institutional review board of Shanghai Chest Hospital (No. KS1716). Because of the study's retrospective nature, the need for written informed consent was waived.

\section{Statistical methods}

OS was measured from RT start to the date of death from any cause or the last follow-up date. Progression-free survival (PFS) was defined as from RT start to the date of tumor progression or the date of death from any cause or the date of the last follow-up. In the OS estimation, patients who received EGFR-TKI or ALK-TKI for progressive diseases during follow-up were censored at the time of TKI initiation. Cox regression analysis was used to detect associations of different factors with PFS and OS. Variables significant on univariate analysis were then entered into multivariate analysis. OS and PFS rates were estimated with the Kaplan-Meier method. The significance of survival estimate differences was tested by the log-rank test. Univariate simple linear regression analysis was performed to find the associations of variables with 1-month post-RT start NLR and PLR. Stepwise multivariate linear regression was performed to assess the influence of variables significant on univariate analysis of 1-month post-RT start NLR and
PLR. Hazard ratios (HRs) and regression coefficient were reported with a $95 \%$ confidence interval (CI). Variables with $\mathrm{P}<0.05$ were considered statistically significant. Statistical analyses were performed with SPSS statistical software, version 19.

\section{Results}

\section{Patient characteristics}

Records for a total of 436 patients with locally advanced NSCLC received a total radiation dose of at least $50 \mathrm{~Gy}$ were identified for this study. Of these patients, 41 were excluded from receiving EGFR-TKI or ALK-TKI as neoadjuvant or adjuvant therapy for RT. Of the remaining 395 patients, 279 patients had both pretreatment and 1 -month post-RT start CBCs. Then 35 patients were excluded owing to the loss of documentation of RT dosimetric data. Finally, 244 patients were included in this study. Baseline characteristics of the patient, tumor, and treatment are listed in Table 1 . The majority of patients were male (87\%) with squamous cell carcinoma (59\%). Tumors were most often T2 (39\%), with N2 (64\%) nodal status. The prescribed radiation dose exceeding or equal to $60 \mathrm{~Gy}$ was used in 206 (84\%) patients. Chemotherapy was given either sequentially or concurrently with RT in 233 (95\%) patients. Eleven (5\%) patients were treated with RT alone due to comorbidities or patient refusal. A total of 115 (47\%) patients received concurrent chemoradiation, and sequential chemoradiation was used in 118 (48\%) patients. The median follow-up time for all patients was 15.5 months (range, 1.9-83.5 months), while for patients who did not experience an event, it was 42.4 months (range, 2.3-81.3 months). The estimated median OS was 15.8 months (95\% CI: 13.9 17.7 months), and the estimated PFS was 8.6 months (95\% CI: 7.1-10.0 months).

\section{Inflammatory biomarkers during $R T$}

During RT, ALC declined each week and generally reached a plateau by the end of RT in all patients. However, the ALC tended to recover 1-month after the completion of RT. Conversely, the NLR and PLR increased each week of RT and also recovered 1-month after the completion of RT (Figure S1). Median baseline ALC, NLR, and PLR were 1,600 cells $/ \mathrm{mm}^{3}, 3.2,155.2$, respectively. Median preRT ALC, NLR, and PLR were 1,600 cells $/ \mathrm{mm}^{3}, 3.2,155.2$, respectively. The median ALC declined to 1,000, 700, 700, 
Table 1 Baseline patient, tumor, and treatment characteristics

\begin{tabular}{|c|c|}
\hline Patient characteristics $(n=244)$ & Median (range or \%) \\
\hline Age, years & $61[22-78]$ \\
\hline \multicolumn{2}{|l|}{ Gender } \\
\hline Male & $213(87)$ \\
\hline Female & $31(13)$ \\
\hline \multicolumn{2}{|l|}{ Family history } \\
\hline No & $226(93)$ \\
\hline Yes & $18(7)$ \\
\hline \multicolumn{2}{|l|}{ ECOG PS } \\
\hline 0 & $20(8)$ \\
\hline 1 & $222(91)$ \\
\hline 2 & $2(1)$ \\
\hline $\mathrm{CCl}$, excluding lung cancer & $2(0-7)$ \\
\hline \multicolumn{2}{|l|}{ Smoking index } \\
\hline$<200$ & $86(35)$ \\
\hline$\geq 200$ & $158(65)$ \\
\hline \multicolumn{2}{|l|}{ Weight loss (6 mon) } \\
\hline$<5 \%$ & $234(96)$ \\
\hline$\geq 5 \%$ & $10(4)$ \\
\hline \multicolumn{2}{|l|}{ Histology } \\
\hline Squamous & $145(59)$ \\
\hline Adenocarcinoma & $77(32)$ \\
\hline NOS & $22(9)$ \\
\hline \multicolumn{2}{|l|}{ Location } \\
\hline Central & $141(58)$ \\
\hline Peripheral & $103(42)$ \\
\hline \multicolumn{2}{|l|}{ Lung lobe } \\
\hline Upper lobe & $153(63)$ \\
\hline
\end{tabular}

Table 1 (continued)

600 , and 500 cells $/ \mathrm{mm}^{3}$ from week 1 to 5 , respectively. The median NLR increased to 4.1, 4.4, 5.6, 6.4, and 6.4 from week 1 to 5 , respectively. The median PLR increased to $196.3,233.4,301.7,308.0$, and 326.7 from week 1 to 5 , respectively. At 1-month after RT completion, the median ALC, NLR, and PLR were 1,000 cells/ $\mathrm{mm}^{3}, 3.8$, and 207.9, respectively.
Table 1 (continued)

\begin{tabular}{|c|c|}
\hline Patient characteristics $(n=244)$ & Median (range or \%) \\
\hline Middle lobe & $35(14)$ \\
\hline Lower lobe & $56(23)$ \\
\hline \multicolumn{2}{|l|}{ Position } \\
\hline Right & $116(48)$ \\
\hline Left & $128(52)$ \\
\hline \multicolumn{2}{|l|}{ T stage } \\
\hline $\mathrm{T} 1$ & $42(17)$ \\
\hline $\mathrm{T} 2$ & $95(39)$ \\
\hline T3 & $46(19)$ \\
\hline $\mathrm{T} 4$ & $61(25)$ \\
\hline \multicolumn{2}{|l|}{ N stag } \\
\hline No & $6(3)$ \\
\hline N1 & $10(4)$ \\
\hline N2 & $157(64)$ \\
\hline N3 & $71(29)$ \\
\hline Gross tumor volume [cc] & $125[10-707]$ \\
\hline \multicolumn{2}{|l|}{ Prescribed dose (Gy) } \\
\hline$<60$ & $38(16)$ \\
\hline$\geq 60$ & $206(84)$ \\
\hline \multicolumn{2}{|l|}{ RT mode } \\
\hline RT alone & $11(5)$ \\
\hline Sequential RT & $118(48)$ \\
\hline Concurrent RT & $115(47)$ \\
\hline Number of chemotherapies & $4[0-18]$ \\
\hline
\end{tabular}

ECOG PS, Eastern Cooperative Oncology Group Performance Status; CCl, Charlson Comorbidity Index; NOS, not otherwise specified; RT, radiotherapy.

\section{Factors that influence patient outcomes}

Factors that influence OS and PFS on univariate and multivariate analyses are summarized in Tables 2 and 3. In univariate analysis, T stage, larger GTV, elevated 1-month post-RT start NLR, elevated 1-month post-RT start PLR, $\mathrm{MBD}$, and both heart and lung dosimetric variables were 
Table 2 Univariate and multivariate analysis of factors potentially associated with PFS

\begin{tabular}{|c|c|c|c|c|c|c|c|c|c|}
\hline \multirow{2}{*}{ Characteristics } & \multicolumn{3}{|c|}{ Univariate } & \multicolumn{3}{|c|}{ Multivariate (model 1 NLR) } & \multicolumn{3}{|c|}{ Multivariate (model 2 PLR) } \\
\hline & $\mathrm{HR}$ & $95 \% \mathrm{Cl}$ & $\mathrm{P}$ & HR & $95 \% \mathrm{Cl}$ & $P$ & HR & $95 \% \mathrm{Cl}$ & $\mathrm{P}$ \\
\hline Age & 0.995 & $0.980-1.011$ & 0.538 & $\mathrm{NI}$ & & & $\mathrm{NI}$ & & \\
\hline ECOG PS & 0.890 & $0.542-1.461$ & 0.645 & $\mathrm{NI}$ & & & & & \\
\hline $\mathrm{CCl} \geq 4$ & 0.660 & $0.325-1.340$ & 0.250 & $\mathrm{NI}$ & & & & & \\
\hline$<200$ & Ref & & & & & & & & \\
\hline$\geq 200$ & 0.829 & $0.618-1.113$ & 0.212 & & & & & & \\
\hline Weight loss $\geq 5 \%$ & 0.827 & $0.407-1.679$ & 0.599 & $\mathrm{NI}$ & & & & & \\
\hline Histology & & & & $\mathrm{NI}$ & & & $\mathrm{NI}$ & & \\
\hline T stage & 1.230 & $1.075-1.408$ & 0.003 & - & - & 0.279 & - & - & 0.137 \\
\hline $\mathrm{N}$ stage & 1.043 & $0.808-1.348$ & 0.745 & $\mathrm{NI}$ & & & $\mathrm{NI}$ & & \\
\hline GTV (cc) & 1.003 & $1.002-1.004$ & $<0.001$ & 1.002 & $1.001-1.003$ & 0.001 & - & - & 0.282 \\
\hline Prescribed dose (Gy) & & & & $\mathrm{NI}$ & & & $\mathrm{NI}$ & & \\
\hline$<60$ & Ref & & & & & & & & \\
\hline$\geq 60$ & 0.821 & $0.565-1.194$ & 0.302 & & & & & & \\
\hline Concurrent & 0.664 & $0.501-0.879$ & 0.004 & 0.649 & $0.490-0.860$ & 0.003 & 0.632 & $0.477-0.838$ & 0.001 \\
\hline Number of chemo & 1.000 & $0.946-1.057$ & 0.995 & $\mathrm{NI}$ & & & & & \\
\hline Baseline ALC & 0.868 & $0.670-1.124$ & 0.283 & $\mathrm{NI}$ & & & $\mathrm{NI}$ & & \\
\hline Baseline PLR & 1.002 & $1.000-1.004$ & 0.052 & $\mathrm{NI}$ & & & - & - & 0.650 \\
\hline 1-month PLR & 1.002 & $1.001-1.002$ & $<0.001$ & $\mathrm{NI}$ & & & 1.001 & $1.000-1.002$ & $<0.001$ \\
\hline 1-month post-RT PLR & 1.000 & $0.999-1.001$ & 0.514 & $\mathrm{NI}$ & & & $\mathrm{NI}$ & & \\
\hline Mean heart dose & 1.000 & $1.000-1.000$ & 0.007 & - & - & 0.075 & - & - & 0.309 \\
\hline Heart V5 (\%) & 1.006 & $1.001-1.010$ & 0.017 & - & - & 0.124 & - & - & 0.396 \\
\hline Heart V20 (\%) & 1.010 & $1.004-1.017$ & 0.002 & - & - & 0.054 & - & - & 0.200 \\
\hline Heart V40 (\%) & 1.012 & $1.003-1.022$ & 0.014 & - & - & 0.142 & - & - & 0.504 \\
\hline Mean lung dose & 1.001 & $1.000-1.001$ & 0.011 & - & - & 0.253 & - & - & 0.633 \\
\hline Lung V5 (\%) & 1.021 & $1.006-1.036$ & 0.007 & - & - & 0.409 & - & - & 0.700 \\
\hline Lung V20 (\%) & 1.044 & $1.014-1.075$ & 0.004 & - & - & 0.111 & - & - & 0.232 \\
\hline Mean body dose & 1.002 & $1.001-1.002$ & $<0.001$ & - & - & 0.081 & 1.001 & $1.001-1.002$ & $<0.001$ \\
\hline
\end{tabular}

PFS, progression-free survival; HR, hazard ratio; Cl, confidence interval; NI, not included in the multivariate model; ECOG PS, Eastern Cooperative Oncology Group Performance Status; CCI, Charlson Comorbidity Index; NOS, not otherwise specified; GTV, gross tumor volume; chemo, chemotherapy; ALC, absolute lymphocyte counts; NLR, neutrophil to lymphocyte ratio; PLR, platelet to lymphocyte ratio; $\mathrm{VX}$, the proportion of volume receiving at least $\mathrm{X}$ Gy. 
Table 3 Univariate and multivariate analysis of factors potentially associated with OS

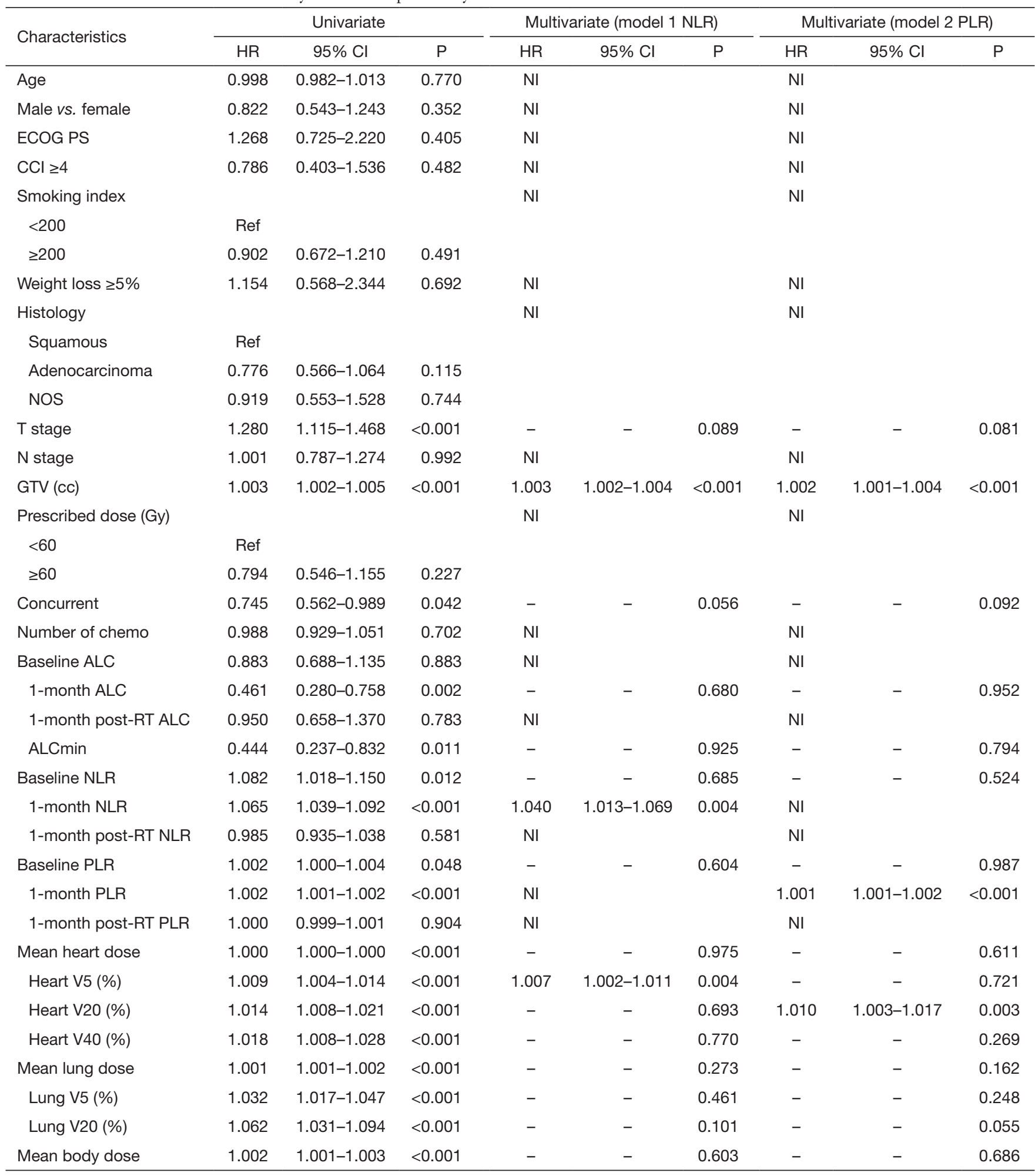

OS, overall survival; HR, hazard ratio; $\mathrm{Cl}$, confidence interval; NI, not included in the multivariate model; ECOG PS, Eastern Cooperative Oncology Group Performance Status; CCI, Charlson Comorbidity Index; NOS, not otherwise specified; GTV, gross tumor volume; chemo, chemotherapy; ALC, absolute lymphocyte counts; NLR, neutrophil to lymphocyte ratio; PLR, platelet to lymphocyte ratio; VX, the proportion of volume receiving at least X Gy. 

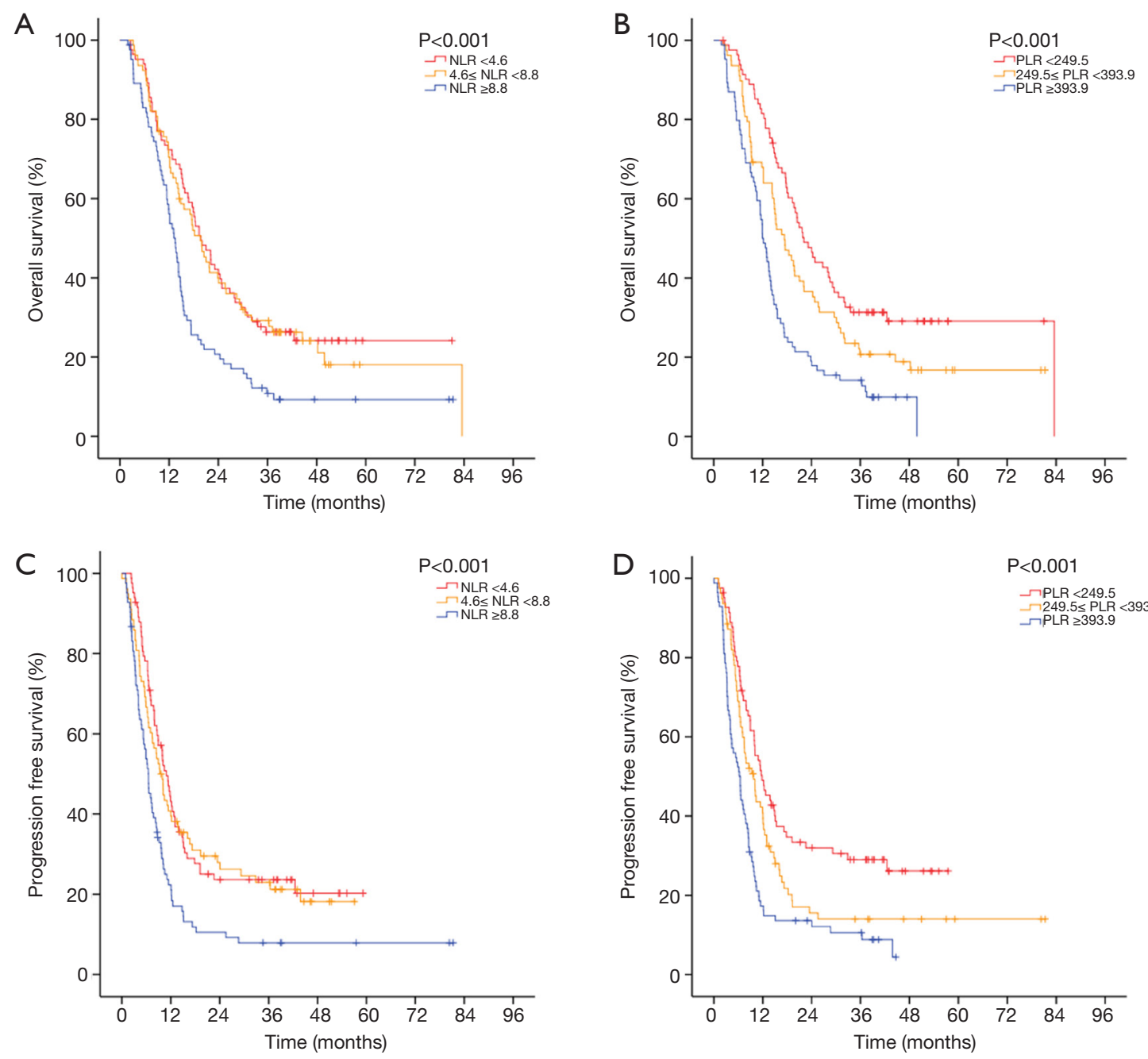

Figure 1 Kaplan-Meier curves for (A,B) OS and (C,D) PFS. (A,C) Patients are stratified by NLR (neutrophil to lymphocyte ratio) at 1-month post-RT start tertiles. (B,D) Patients are stratified by PLR (platelet to lymphocyte ratio) at 1-month post-RT start tertiles. NLR, neutrophil to lymphocyte ratio; PLR, platelet to lymphocyte ratio; OS, overall survival; PFS, progression-free survival.

significantly associated with worse PFS and OS. Also, concurrent chemotherapy and higher 1-month postRT start ALC were significantly associated with better PFS and OS, while ALCmin, baseline NLR, and baseline PLR were only significantly associated with OS. Because NLR and PLR had strong collinearity, they could not be tested simultaneously, so they were tested separately in multivariate analysis.

In the two multivariable modes, higher 1-month postRT start NLR (PFS: HR 1.049, P=0.001; OS: HR 1.003, $\mathrm{P}<0.001)$ and PLR (PFS: HR 1.001, $\mathrm{P}<0.001$; OS: HR 1.001, $\mathrm{P}<0.001)$ were independent prognostic factors for worse PFS and OS. Also, larger GTV and increasing MBD were significantly associated with worse PFS $(\mathrm{P}<0.05)$, and larger GTV and increasing heart V5 or heart V20 were significantly associated with worse $\mathrm{OS}(\mathrm{P}<0.05)$. Survival curves are shown stratified into tertiles to illustrate the effects of 1-month post-RT start NLR and PLR on OS and PFS. The OS and PFS progressively diminished with higher NLR and PLR, respectively (Figure 1).

\section{Factors associated with post-RT NLR and PLR}

The results of univariate and multivariate linear regression associating factors with 1-month post-RT start NLR and PLR are summarized in Tables 4 and 5. T stage, $\log _{10}$ (GTV), 
Table 4 Univariate and multivariate linear regression associating variables with 1-month NLR

\begin{tabular}{|c|c|c|c|c|c|c|}
\hline Characteristics & \multicolumn{2}{|c|}{ Univariate } & $\mathrm{P}$ & \multicolumn{3}{|c|}{ Multivariate } \\
\hline Age & - & - & 0.122 & $\mathrm{NI}$ & & \\
\hline Male vs. female & - & - & 0.490 & $\mathrm{NI}$ & & \\
\hline $\mathrm{CCl} \geq 4$ & - & - & 0.584 & $\mathrm{NI}$ & & \\
\hline $\mathrm{N}$ stage & - & - & 0.990 & $\mathrm{NI}$ & & \\
\hline $\log _{10}$ GTV & 5.427 & $3.524-7.330$ & $<0.001$ & - & - & 0.084 \\
\hline Prescribed dose (Gy) & - & - & 0.457 & $\mathrm{NI}$ & & \\
\hline Concurrent & - & - & 0.741 & $\mathrm{NI}$ & & \\
\hline Heart V5 (\%) & 0.043 & $0.021-0.065$ & $<0.001$ & - & - & 0.127 \\
\hline Heart V20 (\%) & 0.066 & $0.035-0.097$ & $<0.001$ & 0.150 & $0.082-0.218$ & $<0.001$ \\
\hline Heart V40 (\%) & 0.065 & $0.016-0.115$ & 0.010 & -0.202 & $-0.308-0.096$ & $<0.001$ \\
\hline Mean lung dose & 0.004 & $0.001-0.006$ & 0.004 & - & - & 0.602 \\
\hline Lung V5 (\%) & 0.135 & $0.071-0.199$ & $<0.001$ & - & - & 0.591 \\
\hline Lung V20 (\%) & 0.181 & $0.055-0.308$ & 0.005 & - & - & 0.992 \\
\hline Mean body dose & 0.010 & $0.007-0.013$ & $<0.001$ & 0.008 & $0.005-0.012$ & $<0.001$ \\
\hline
\end{tabular}

$\mathrm{NLR}$, neutrophil to lymphocyte ratio; $\beta$, standardized coefficient; $\mathrm{Cl}$, confidence interval; $\mathrm{NI}$, not included in the multivariate model; CCI, Charlson Comorbidity Index; GTV, gross tumor volume; VX, the proportion of volume receiving at least X Gy.

baseline NLR, MBD, and heart and lung dosimetric variables were significantly associated with 1 -month postRT start NLR on univariate simple linear regression. However, stepwise multivariate linear regression identified only 4 factors significantly associated with 1 -month postRT start NLR: baseline NLR $(\beta=0.655, \mathrm{P}<0.001)$, heart V20 $(\beta=0.150, P<0.001)$, heart V40 $(\beta=-0.202, P<0.001)$, and $\operatorname{MBD}(\beta=0.008, P<0.001)$. T stage, $\log _{10}(\mathrm{GTV})$, baseline PLR, and heart and lung dosimetric variables were significantly associated with 1-month post-RT start PLR on univariate simple linear regression. With multiple stepwise linear regression analysis, baseline PLR $(\beta=0.975, \mathrm{P}<0.001)$ and MBD $(\beta=0.407, \mathrm{P}<0.001)$ were significant factors associated with 1-month post-RT start PLR.

\section{Subgroup analysis of patients receiving $R T$ without concurrent chemotherapy}

Because chemotherapy can also affect the NLR and PLR, we further performed a subgroup analysis of patients receiving RT without concurrent chemotherapy. In multivariate cox analysis, 1-month post-RT start NLR $(\mathrm{P}<0.05)$ and PLR $(\mathrm{P}<0.05)$ were still independent prognostic factors for PFS and OS (Tables $S 1, S 2)$. In multivariate linear regression analysis, baseline count $(\mathrm{P}<0.001)$ and $\mathrm{MBD}(\mathrm{P}<0.001)$ were significantly associated with 1-month post-RT start NLR and PLR (Tables S3,S4).

\section{Discussion}

Our study demonstrated that radiation for LA-NSCLC could dramatically reduce the lymphocyte count and increase the NLR and PLR during RT. Higher 1-month post-RT start NLR or PLR, but not baseline or 1-month post-RT, was correlated with worse PFS and OS. This relationship held on multivariate analysis when controlling for common patient demographic, treatment, and tumor characteristics. Multiple stepwise regression analysis 
Table 5 Univariate and multivariate linear regression associating variables with 1-month PLR

\begin{tabular}{|c|c|c|c|c|c|c|}
\hline Characteristics & \multicolumn{3}{|c|}{ Univariate } & \multicolumn{3}{|c|}{ Multivariate } \\
\hline Age & - & - & 0.622 & $\mathrm{NI}$ & & \\
\hline Male vs. female & - & - & 0.649 & $\mathrm{NI}$ & & \\
\hline $\mathrm{CCl} \geq 4$ & - & - & 0.789 & $\mathrm{NI}$ & & \\
\hline $\mathrm{N}$ stage & - & - & 0.838 & $\mathrm{NI}$ & & \\
\hline $\log _{10}$ GTV & 252.969 & $174.562-331.375$ & $<0.001$ & - & - & 0.534 \\
\hline Prescribed dose (Gy) & - & - & 0.930 & $\mathrm{NI}$ & & \\
\hline Concurrent & - & - & 0.430 & $\mathrm{NI}$ & & \\
\hline Heart V5 (\%) & 1.571 & $0.653-2.489$ & 0.001 & - & - & 0.852 \\
\hline Heart V20 (\%) & 2.312 & $0.987-3.636$ & 0.001 & - & - & 0.813 \\
\hline Heart V40 (\%) & 2.726 & $0.648-4.803$ & 0.010 & - & - & 0.795 \\
\hline Mean lung dose & 0.119 & $0.018-0.220$ & 0.022 & - & - & 0.638 \\
\hline Lung V5 (\%) & 4.147 & $1.431-6.863$ & 0.003 & - & - & 0.704 \\
\hline Lung V20 (\%) & 5.494 & $0.158-10.829$ & 0.044 & - & - & 0.693 \\
\hline Mean body dose & 0.446 & $0.315-0.578$ & $<0.001$ & 0.407 & $0.284-0.530$ & $<0.001$ \\
\hline
\end{tabular}

PLR, platelet to lymphocyte ratio; $\beta$, standardized coefficient; $\mathrm{Cl}$, confidence interval; $\mathrm{NI}$, not included in the multivariate model; CCI, Charlson Comorbidity Index; GTV, gross tumor volume; VX, the proportion of volume receiving at least X Gy.

confirmed that baseline NLR, heart V20, V40, and MBD were correlated significantly with 1 -month post-RT start NLR. Also, baseline PLR and MBD were significantly associated with 1 -month post-RT start PLR. Subgroup analysis of patients receiving RT without concurrent chemotherapy also demonstrated that 1 -month post-RT start NLR and PLR were independent prognostic factors for PFS and OS. Baseline count and MBD were also correlated with 1-month post-RT start NLR and PLR.

Accumulating evidence shows that inflammation and immune response are essential stimulators for cancer initiation, progression, and prognosis $(19,20)$. Lymphocytes could inhibit tumor cell proliferation and migration by secreting various cytokines (21). However, research has indicated that neutrophils induced by transforming growth factor- $\beta$ (TGF- $\beta$ ) dominate the immune cell composition in NSCLC and influence the cytolytic activity of lymphocytes or natural killer cells $(22,23)$. Thus, increased neutrophil counts are associated with a poor prognosis. It has been reported that platelets could release vascular endothelial growth factor (VEGF) and other cytokines to activate angiogenesis, associated with tumor metastasis and poor prognosis (24). There have been numerous studies on the prognostic potential of NLR and PLR in various solid tumors. Two meta-analyses have found that high NLR and PLR are associated with worse OS and PFS in NSCLC of different stages and treatment $(13,25)$. Our study also revealed that patients with elevated NLR or PLR were more likely to have worse OS and PFS.

Baseline count, heart V20, V40, or MBD was significantly associated with 1 -month post-RT start NLR or PLR in our cohort. Similarly, other studies have identified that heart V50 $>25 \%$ were significantly associated with an NLR $>10.54$ months post-RT (26). Tang et al. demonstrated that a higher volume of lung tissue receiving $5 \mathrm{~Gy}$ and higher GTV were significant predictors of lymphocyte nadir (9). Peripheral lymphocytes are known to be the most radiosensitive cells despite mitotic inactivity with a D50 as 
low as 2 Gy (27). Yovino et al. found that a single radiation fraction delivered 0.5 Gy exposure to $5 \%$ of circulating cells. What is more, after the course of 30 fractions, $99 \%$ of circulating cells would receive more than 0.5 Gy (28). Likewise, we found that after 18-30 fractions of radiation, ALC tended to decline. Others found that reducing RT treatment volumes in glioblastoma was associated with less lymphopenia (29). Research has also demonstrated that the integral body dose was negatively correlated with post-treatment ALC (30). Therefore we could reasonably extrapolate that the use of SBRT or proton may minimize the heart dose and body dose, reducing the adverse effect of radiation on NLR or PLR.

The results of the PACIFIC trial show that treatment with adjuvant immunotherapy following definitive chemoradiation results in a significant improvement in PFS and OS for LA-NSCLC (5). This trial warrants further prospective research on the anti-tumor immune response after radical RT. Retrospective studies have demonstrated that elevated pretreatment NLR and PLR are associated with shorter OS and PFS and with lower response rates in patients with metastatic NSCLC treated with nivolumab independently of other prognostic factors (31). Also, it has been shown that increased NLR and PLR values 6 weeks after baseline are associated with shorter OS and PFS in patients who have advanced cancer treated with immunotherapy (32). While our findings were in agreement with these previous studies, we demonstrated for the first time that increased 1-month post-RT start NLR and PLR are associated with worse PFS and OS.

There are several limitations to this study. First, this was a retrospective study in a single tertiary academic center with selection bias and loss of laboratory value measurements. Therefore, a large sample multicenter clinical study or external validation should be performed in the future. Also, we only studied the prognostic association between baseline, 1-month post-RT start, or 1-month postRT completion inflammatory biomarkers with clinical outcomes. Other variables that illustrate more delicate descriptions of inflammatory biomarkers change, including subpopulations, should be investigated and might be more predictive.

\section{Conclusions}

In this study, we have demonstrated for the first time a significant association between heart V20, V40 or MBD and 1-month post-RT start NLR or PLR, in addition to confirming the prognostic effect of 1 -month post-RT start NLR and PLR on multivariate analysis in advanced NSCLC treated with definitive RT, taking into account the mutation of EGFR and ALK rearrangements.

\section{Acknowledgments}

Funding: This study was supported by the National Key Research and Development Program of China (2016YFC0905502), the Shanghai Shen Kang Hospital Development Center Clinical Research Plan of SHDC (16CR1016A), and the Project of Shanghai Science and Technology Commission (18YF1421500).

\section{Footnote}

Reporting Checklist: The authors have completed the STROBE reporting checklist. Available at http://dx.doi. org/10.21037/tlcr-20-831

Data Sharing Statement: Available at http://dx.doi. org/10.21037/tlcr-20-831

Conflicts of Interest: All authors have completed the ICMJE uniform disclosure form (available at http://dx.doi. org/10.21037/tlcr-20-831). The authors report grants from the National Key Research and Development Program of China (2016YFC0905502), grants from the Shanghai Shen Kang Hospital Development Center Clinical Research Plan of SHDC (16CR1016A), grants from the Project of Shanghai Science and Technology Commission (18YF1421500), during the conduct of the study.

Ethical Statement: The authors are accountable for all aspects of the work in ensuring that questions related to the accuracy or integrity of any part of the work are appropriately investigated and resolved. All procedures performed in this study involving human participants were in accordance with the Declaration of Helsinki (as revised in 2013). The study was approved by the institutional review board of Shanghai Chest Hospital (No. KS1716). Because of the study's retrospective nature, the need for written informed consent was waived.

Open Access Statement: This is an Open Access article distributed in accordance with the Creative Commons Attribution-NonCommercial-NoDerivs 4.0 International License (CC BY-NC-ND 4.0), which permits the non- 
commercial replication and distribution of the article with the strict proviso that no changes or edits are made and the original work is properly cited (including links to both the formal publication through the relevant DOI and the license). See: https://creativecommons.org/licenses/by-nc-nd/4.0/.

\section{References}

1. Stinchcombe TE, Zhang Y, Vokes EE, et al. Pooled Analysis of Individual Patient Data on Concurrent Chemoradiotherapy for Stage III Non-Small-Cell Lung Cancer in Elderly Patients Compared With Younger Patients Who Participated in US National Cancer Institute Cooperative Group Studies. J Clin Oncol 2017;35:2885-92.

2. Abbas MN, Ayoola A, Padman S, et al. Survival and late toxicities following concurrent chemo-radiotherapy for locally advanced stage III non-small cell lung cancer: findings of a 10-year Australian single centre experience with long term clinical follow up. J Thorac Dis 2019;11:4241-8.

3. Ramnath N, Dilling TJ, Harris LJ, et al. Treatment of stage III non-small cell lung cancer: Diagnosis and management of lung cancer, 3rd ed: American College of Chest Physicians evidence-based clinical practice guidelines. Chest 2013;143:e314S-e40S.

4. Waddle MR, Ko S, Johnson MM, et al. Post-operative radiation therapy in locally advanced non-small cell lung cancer and the impact of sequential versus concurrent chemotherapy. Transl Lung Cancer Res 2018;7:S171-5.

5. Antonia SJ, Villegas A, Daniel D, et al. Overall Survival with Durvalumab after Chemoradiotherapy in Stage III NSCLC. N Engl J Med 2018;379:2342-50.

6. Gray JE, Villegas AE, Daniel DB, et al. Three-year overall survival update from the PACIFIC trial. American Society of Clinical Oncology; 2019.

7. Proctor MJ, Morrison DS, Talwar D, et al. An inflammation-based prognostic score (mGPS) predicts cancer survival independent of tumour site: a Glasgow Inflammation Outcome Study. Br J Cancer 2011;104:726-34.

8. Fernandes JV, Cobucci RN, Jatoba CA, et al. The role of the mediators of inflammation in cancer development. Pathol Oncol Res 2015;21:527-34.

9. Tang C, Liao Z, Gomez D, et al. Lymphopenia association with gross tumor volume and lung V5 and its effects on non-small cell lung cancer patient outcomes. Int J Radiat Oncol Biol Phys 2014;89:1084-91.
10. Templeton AJ, McNamara MG, Seruga B, et al. Prognostic role of neutrophil-to-lymphocyte ratio in solid tumors: a systematic review and meta-analysis. J Natl Cancer Inst 2014;106:dju124.

11. Kinoshita A, Onoda H, Imai N, et al. Comparison of the prognostic value of inflammation-based prognostic scores in patients with hepatocellular carcinoma. Br J Cancer 2012;107:988-93.

12. Kang KH, Efird JT, Sharma N, et al. Prognostic potential of neutrophil-to-lymphocyte ratio and lymphocyte nadir in stage III non-small-cell lung cancer. Future Oncol 2017;13:1405-14.

13. Gu X, Sun S, Gao XS, et al. Prognostic value of platelet to lymphocyte ratio in non-small cell lung cancer: evidence from 3,430 patients. Sci Rep 2016;6:23893.

14. Song X, Chen D, Yuan M, et al. Total lymphocyte count, neutrophil-lymphocyte ratio, and platelet-lymphocyte ratio as prognostic factors in advanced non-small cell lung cancer with chemoradiotherapy. Cancer Manag Res 2018;10:6677-83.

15. Sellins KS, Cohen JJ. Gene induction by gammairradiation leads to DNA fragmentation in lymphocytes. J Immunol 1987;139:3199-206.

16. Campian JL, Sarai G, Ye X, et al. Association between severe treatment-related lymphopenia and progressionfree survival in patients with newly diagnosed squamous cell head and neck cancer. Head Neck 2014;36:1747-53.

17. Wang X, Lu J, Teng F, et al. Lymphopenia association with accelerated hyperfractionation and its effects on limitedstage small cell lung cancer patients' clinical outcomes. Ann Transl Med 2019;7:385.

18. Fang P, Jiang W, Davuluri R, et al. High lymphocyte count during neoadjuvant chemoradiotherapy is associated with improved pathologic complete response in esophageal cancer. Radiother Oncol 2018;128:584-90.

19. Mantovani A, Allavena P, Sica A, et al. Cancer-related inflammation. Nature 2008;454:436-44.

20. Hanahan D, Weinberg RA. Hallmarks of cancer: the next generation. Cell 2011;144:646-74.

21. Coussens LM, Werb Z. Inflammation and cancer. Nature 2002;420:860-7.

22. Pillay J, Kamp VM, van Hoffen E, et al. A subset of neutrophils in human systemic inflammation inhibits T cell responses through Mac-1. J Clin Invest 2012;122:327-36.

23. Kargl J, Busch SE, Yang GH, et al. Neutrophils dominate the immune cell composition in non-small cell lung cancer. Nat Commun 2017;8:14381. 
24. Bambace NM, Holmes CE. The platelet contribution to cancer progression. J Thromb Haemost 2011;9:237-49.

25. Gu XB, Tian T, Tian XJ, et al. Prognostic significance of neutrophil-to-lymphocyte ratio in non-small cell lung cancer: a meta-analysis. Sci Rep 2015;5:12493.

26. Contreras JA, Lin AJ, Weiner A, et al. Cardiac dose is associated with immunosuppression and poor survival in locally advanced non-small cell lung cancer. Radiother Oncol 2018;128:498-504.

27. Yovino S, Grossman SA. Severity, etiology and possible consequences of treatment-related lymphopenia in patients with newly diagnosed high-grade gliomas. CNS Oncol 2012;1:149-54.

28. Yovino S, Kleinberg L, Grossman SA, et al. The etiology of treatment-related lymphopenia in patients with malignant gliomas: modeling radiation dose to circulating lymphocytes explains clinical observations and suggests methods of modifying the impact of radiation on immune cells. Cancer Invest 2013;31:140-4.

Cite this article as: $\mathrm{Xia}$ WY, Zhu XR, Feng W, Liu J, Wang JM, Lv CX, Zhang Q, Yu W, Cai XW, Fu XL. Neutrophillymphocyte ratio and platelet-lymphocyte ratio associations with heart and body dose and their effects on patient outcomes in locally advanced non-small cell lung cancer treated with definitive radiotherapy. Transl Lung Cancer Res 2020;9(5):1996-2007. doi: 10.21037/tlcr-20-831
29. Rudra S, Hui C, Rao YJ, et al. Effect of Radiation Treatment Volume Reduction on Lymphopenia in Patients Receiving Chemoradiotherapy for Glioblastoma. Int J Radiat Oncol Biol Phys 2018;101:217-25.

30. Joseph N, McWilliam A, Kennedy J, et al. Post-treatment lymphocytopaenia, integral body dose and overall survival in lung cancer patients treated with radical radiotherapy. Radiother Oncol 2019;135:115-9.

31. Diem S, Schmid S, Krapf M, et al. Neutrophil-toLymphocyte ratio (NLR) and Platelet-to-Lymphocyte ratio (PLR) as prognostic markers in patients with nonsmall cell lung cancer (NSCLC) treated with nivolumab. Lung Cancer 2017;111:176-81.

32. Bilen MA, Martini DJ, Liu Y, et al. The prognostic and predictive impact of inflammatory biomarkers in patients who have advanced-stage cancer treated with immunotherapy. Cancer 2019;125:127-34.

(English Language Editors: J. Brown and J. Chapnick) 